\title{
Cell biology: tracking a cell's cycle
}

\author{
Vivien Marx
}

The tools that clock a cell's everyday affairs reveal plenty that's out of the ordinary.

A cell's DNA condenses at certain times in visible ways, but whether unlabeled cells are undergoing mitosis can be hard to discern. Cells might be in G1, when protein production ramps up; $\mathrm{S}$, when DNA is replicated; G2, when DNA quality control takes place; or $\mathrm{M}$, when mitosis occurs and a cell finalizes its symmetric or asymmetric split into two cells. These events are central to biological events ranging from development and differentiation to cancer.

In 1882, German biologist Walther Flemming drew detailed illustrations of mitosis and chromatin structure within single cells, and "it's fascinating that we continue to uncover additional layers of regulation over a hundred years later with advances in technology such as single cell genomics," note Sarah Teichmann, a researcher at the Wellcome Trust Sanger Institute, and Kedar Natarajan, a postdoctoral fellow in her lab, in a joint e-mail. It intrigues them, for example, that cells continue transcription during G1, $\mathrm{S}$ and G2 and can keep responding to environmental cues and signaling related to differentiation.

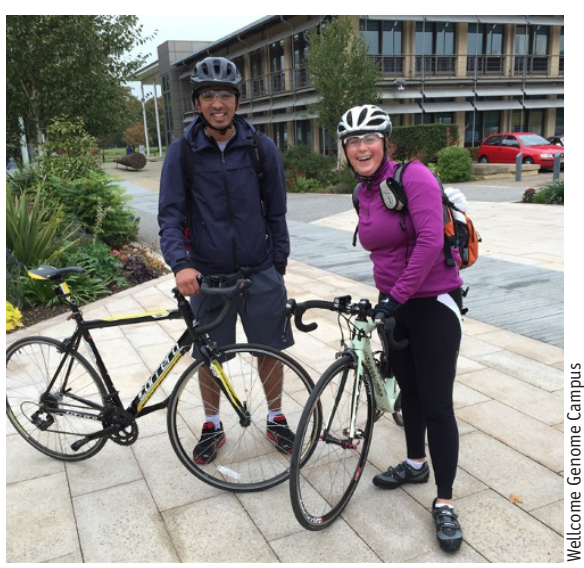

Sarah Teichmann (right) and Kedar Natarajan (left) cycle together to talk about the cell cycle.
In 2008, Atsushi Miyawaki and his team at RIKEN in Japan presented a genetically encoded method to deliver a visual readout of cell cycles ${ }^{1}$. Robert Newman and Jin Zhang, at the Johns Hopkins University School of Medicine, described the approach as "street lights on the road to mitosis." These 'street lights' are provided by fluorescence ubiquitination-based cell-cycle indicator (Fucci) - a technology that harnesses the way cellular proteins oscillate during the cell cycle.

With the Fucci system, as Miyawaki explains, cells in G1 phase fluoresce red owing to a fluorescent protein hooked onto 91 amino acids of the ubiquitination domain of the human protein Cdt1. As S phase begins, the Cdt 1 construct begins to be degraded and another comes into play, a fluorescent protein tacked onto 110 amino acids of the ubiquitination domain of the protein Geminin. This reporter lets cells in S and G2 phase fluoresce green. During M phase, fluorescence begins to fade as the green reporter is degraded and the red reporter is not yet being produced. In 2014, the team in Japan presented mVenus-p27K-, an indicator for identifying cells in G0 phase, when they leave the cell cycle either temporarily or permanently and are quiescent ${ }^{2}$.

In 2016, Michael Lin and his team at Stanford University developed Fucci4, which reports simultaneously on all four cell-cycle phases in live cells ${ }^{3}$. To track kinases in neurons, Lin had worked on fluorescence resonance energy transfer (FRET) reporters and fluorescent protein engineering as a postdoctoral fellow in Roger Tsien's lab at the University of California, San Diego. After learning about Fucci, he realized he could use fluorescent protein wavelength engineering to return to his "first scientific love of the cell cycle." Lin and his team made Fucci4 by adding a reporter for the S-to-G2 transition to 'classic Fucci' using their new far-red

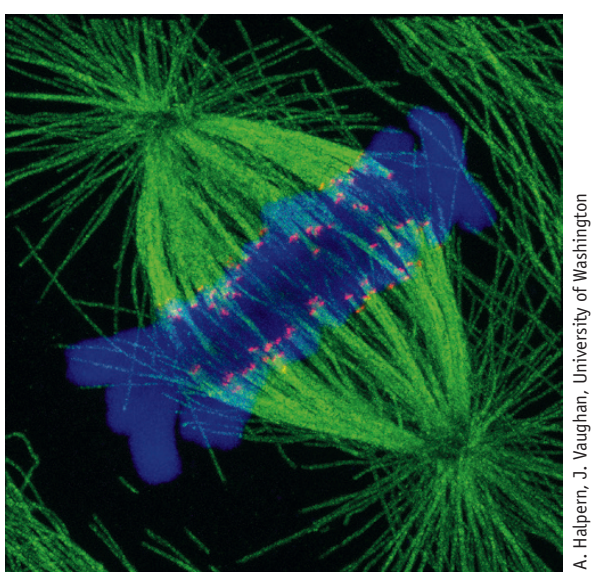

A dividing PtK1 cell from a rat, showing tubulin (green), a kinetochore protein (red) and DNA (blue). Although mitosis is well studied, labs are finding out ever more about it.

fluorescent protein mMaroon1, which helps to visualize chromatin condensation that typically accompanies mitosis.

These indicators enable researchers to explore the cell cycle expansively. Titia de Lange and her team at the Rockefeller University used Fucci and live-cell imaging to reveal that telomere-driven genome instability is a key factor in how tetraploidization occurs in cancer cells. Dysfunctional telomeres can cause cells to bypass mitosis and re-enter S phase, which leads to cells with four copies of the genome rather than two.

In developmental biology, Shigetomo Fukuhara, previously at the National Cerebral and Cardiovascular Center in Japan and now at Nippon Medical School, wanted to study how vascular structures develop and grow in different parts of zebrafish. He and his team imaged endothelial cells to track cellcycle progression in the developing zebrafish embryo. To observe what was previously possible to see only with tissue staining, the team developed a transgenic zebrafish line that 


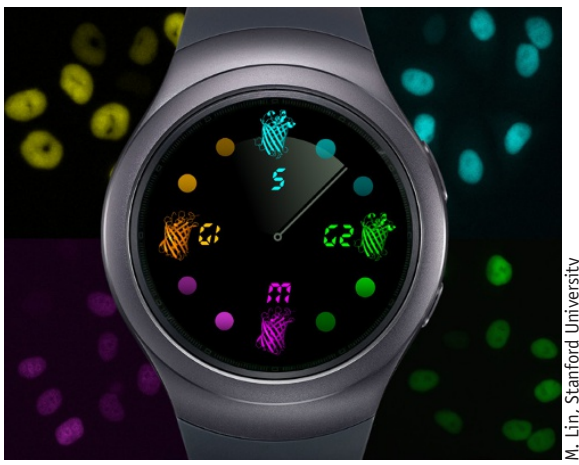

Fucci4, developed at Stanford University, reports simultaneously on the four cell-cycle phases in live cells.

expresses Fucci (zFucci) in endothelial cells. The researchers validated their observations by labeling endothelial cells in S phase with 5-ethynyl-2'-deoxyuridine (EdU), using time-lapse imaging and looking at the effects of cell-cycle inhibitors on these cells.

Ignoring the cell cycle can trip up investigators. Genetic or chemical perturbation of a cell that has a cell-cycle-dependent phenotype might shift the cell-cycle phase without affecting the phenotype. That can lead to misinterpretation of the results, says Prisca Liberali, a researcher at Friedrich Miescher Institute for Biomedical Research. Other potential pitfalls are related to cell signaling. Liberali and her colleagues have shown that G2 cells have higher activation of the signaling kinases AKT and ERK than cells in other phases. In a screen, however, a researcher might score results and not take the numbers of cells in G1 versus G2 into account, she says. And when exploring cellto-cell variability, the cell cycle is also best not ignored. "Clearly, not accounting for differences in cell-cycle stages means ignoring a strong deterministic source of variability," she says.

\section{Going deep}

To explore the mechanisms of cancer drugs in vivo, Stefan Florian, a postdoctoral fellow in Tim Mitchison's lab at Harvard Medical School, has used Fucci, which he calls "a very ingenious method." Before Fucci4, he and his colleagues found a way to distinguish three cell-cycle phases in a live anesthetized mouse using two Fucci indicators and a labeled histone as a chromosome morphology cue ${ }^{4}$. He got along using three markers but he looks forward to trying Fucci4, because it offers the most information, he says. And the G0/ G1 sensor will be "really interesting," he says, if it works as advertised.

Even with three colors, Florian needed a computational tool to discern the densely packed cells in $3 \mathrm{D}$ culture. During imaging, he repeatedly asked himself: "Have I looked at this already or not? Did I count this or didn't I count this?" To complete the project he co-developed a computational tool to quantitatively assess cell-cycle states.

Florian uses both intravital imaging and $3 \mathrm{D}$ culture systems, which give him more time than the few hours he has when working with an anesthetized mouse. Generally speaking, mammalian cells growing in vitro complete the cell cycle in 24 to 48 hours. Working with his $3 \mathrm{D}$ system allows him to see whether cells went through mitosis after exposure to a compound. Cells growing in $3 \mathrm{D}$, such as in hanging droplets of medium, also behave more like cells in their natural environment than when growing on a flat dish.

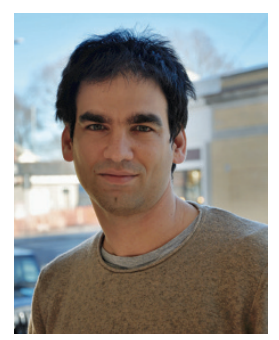

Stefan Florian computational tool to quantitatively assess cell-cycle states. co-developed a

With 3D culture and Fucci, "you get longitudinal information and you can say something about the dynamics of the process," says Florian. The data can be validated with disassociated tissue and fluorescence-activated cell sorting (FACS) or with tissue slices and antibody staining. With 3D culture, however, he faces some challenges with Fucci because with a deeper gaze, the fluorescence dims. Using one color an experimenter can ask if it is green or not, but more colors make distinctions tougher, he says. "It's very hard to tell-if you have a deep cell-is it dim because it's deep or is it dim because it's changing the cell-cycle phase?"

To address this type of challenge, Miyawaki thinks two-photon microscopy will be helpful. For fixed 3D samples, tissue clearing methods, including the Scale technique developed in his lab, are advisable. Lin recommends confocal microscopy and, if it is possible to illuminate the sample from the side, selective plane illumination microscopy. Two-photon microscopy is not preferable, he says, because it calls for multiple precise excitation wavelengths. "One can make corrections for loss of light with increasing depth by testing the imaging system with the fluorophore present at the same concentration at different depths, but this shouldn't be necessary if time lapse is being performed and if one can see the rise and fall of each marker," says Lin.

\section{Solving other hindrances}

Another issue Florian encounters is inhomogeneous expression of fluorescent proteins. As Lin explains, he and his team put the two constructs of the original Fucci system in one lentivirus and the two new Fucci4 components in another. Lin's team did not need homogenous expression for their experiments, so they didn't perform selections for all four constructs. But "one could imagine selecting for both viruses using FACS, even perhaps gating for different relative intensities at the various wavelengths, so one can determine which intensities give the maximal signal without affecting the cell," he says.

Miyawaki, too, recommends lentiviral vectors to enable efficient transduction and recommends creating vectors with multiple relevant loci on a single construct, for example by concatenating the Fucci probes via the $2 \mathrm{~A}$ peptide to obtain bicistronic Fucci variants. This approach was developed to address issues, such as transgene inactivation, that labs encountered in mouse models incorporating Fucci.

Frequently, researchers contact the Fucci developers at RIKEN with concerns about the indicators' effects on cell physiology. These fears, says Miyawaki, are stoked by lax probe-naming habits in the research community. He and his team went to great pains to identify the domain that is both necessary and sufficient for cell-cycle probing and that does not affect cell-cycle progression in the cells receiving these genetic constructs. "First, we constructed numerous constructs with different truncations and mutations," says Miyawaki. "Second, we prepared stable transformant cells for each construct." They did extended time-lapse imaging experiments to see how well the sensors helped to distinguish cell-cycle phases. Probe names such as mKO2-Cdt1 and mAG-Geminin, which do not specify whether the proteins used are full length or fragments, are "definitely misleading," he says. A probe that uses the entire Cdt1 or Geminin protein instead of a select, targeted number of amino acids, he says, would deeply affect cell-cycle progression. It would be useful, he says, to include the full names of probes, such as $\mathrm{mKO} 2$-hCdt1(30/120) and mAG-hGem(1/110), in a paper's footnotes or methods section. 


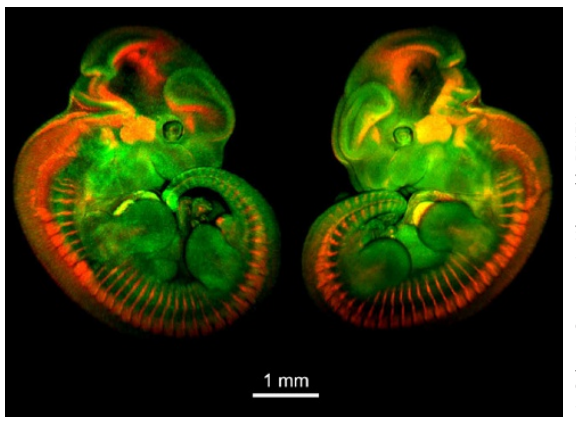

Fucci in mouse embryos. The technology harnesses the oscillation of proteins during the cell cycle. Green signal indicates proliferation, and red signal indicates differentiation. This tissue was cleared with ScaleA2.

Fucci has many strengths in research with live cells, says Philipp Kaldis, a researcher at the Institute of Molecular and Cell Biology in Singapore. It can, however, be tricky to get plasmids into primary cells. Fucci helps researchers determine cell-cycle phases, but distinguishing the transitions is more complicated. Kaldis says that FACS can work well and could become a reliable way to track cells through the cell cycle as the methods grow more sophisticated. He prefers studying biological processes in vivo in mice. Kaldis uses knockout mice that lack cyclin-dependent kinases (CDKs) or other cell-cycle regulators to identify proteins that are dephosphorylated during the cell cycle. But he still runs into issues: G2 is not always $\mathrm{G} 2$, for example. In the Cdk1-knockout mouse, cells will arrest in G2 and then, after a delay, start endoreplication, in which the genome replicates without cell division. "This situation is quite different from a cell in G2 that does not endoreplicate," he says.

Experimentally synchronizing the cell cycle can help researchers. T cells can be isolated and stimulated to enter the cell cycle synchronously, says Kaldis. In the adult liver, hepatocytes are quiescent, but at an injury site, cells enter the cell cycle in a synchronized fashion and become "facultative stem cells," he says. "This is a great system to study cell-cycle regulation in the mouse." He combines this with magnetic resonance imaging and intravital imaging via a glass window implanted over a small injury in the liver and tracks the cell cycle using two-photon microscopy and autofluorescence. Syncing the cell cycle is still a type of perturbation, so "single-cell analysis of an unperturbed population is the best way to go," says Kaldis, referring to Fucci and cell sorting.

\section{Compute these cells}

A number of computational tools have been developed for single-cell cell-cycle analy$\operatorname{sis}^{5-7}$. Some use single-cell gene expression data, others use cell imaging data. Single-cell RNA-seq (scRNA-seq) is a way to zoom in and identify and distinguish cell-states within a population on the basis of gene expression. Along with colleagues, the Sanger Institute's Teichmann co-developed a single-cell latent variable model (scLVM) and Cyclone package that applies machine learning and statistical methods to assign cell-cycle stages from scRNA-seq data and identify the contribution of cell-cycle stage to gene expression signatures.

Teichmann and Natarajan explain that they initially used this approach on mouse embryonic stem cells to distinguish key immune-cell driver genes during differentiation of naive T lymphocytes to specialized type 2 helper T cells. During this process, cells respond to differentiation signals while also proliferating. Gene expression is affected by the differentiation signal, cell-cycle-based gene expression and the combination of differentiation cues and the cell cycle, all to varying degrees. The researchers are now using their tools to study cell-cycle influences on gene expression and cell-fate decisions and to discover new cell-cycle regulators.

The team is using Fucci indicators and variants in their experiments. Given that the reporters provide a more precise separation of cell-cycle stages, the researchers hope the work will provide a higher-resolution view of gene expression states of cells across the cell cycle. Perhaps it will also help assign and deconvolute stage-specific expression. The higher resolution will especially aid when validating new cell-cycle gene candidates and their stage-specific regulation, they say. The approach will provide training sets for new computational methods, potentially with better cell-cycle stage assignment capability, that can better distinguish between cell-cycle rates in different sets of single cells.

Another tool, Cycler, developed by Liberali and colleagues, works with imaging data from fixed, stained cells and can help researchers analyze the activities of individual mammalian cells in a population as they go through the cell cycle. Liberali says she and her colleagues plan to use this tool for various types of live-cell imaging with Fucci and other cell-cycle reporters.

Cycler orders cells on the basis of their position in the cell cycle. The tool is underpinned by an algorithm, called Wanderlust, that was originally developed for single-cell cytometry data. For validation, the team used ERK signaling peaks with FRET reporting. Cycler could help bring added resolution for labs working with scRNA-seq data, which lack the morphological cues from imaging, the Cycler developers say. Better resolution is needed because changes in gene expression due to external or developmental cues can be confounded with changes due to cell cycle.

Another tool is Oscope, a statistically based approach developed at the University of Wisconsin-Madison that clusters groups of oscillatory signals in scRNA-seq data. It, too, addresses the issues labs can face with scRNA-seq data, such as missing or misrepresenting gene expression oscillations that typically accompany the cell cycle. Oscope constructs the 'cyclic order' of samples for each group of oscillating genes in order to capture the cell-cycle state. It cannot, however, be applied to longitudinal studies. To validate Oscope and the cell-cycle profiles it discovered, the developers used Fucci-labeled H1 human embryonic stem cells to identify cells in G1, S or G2/M. Then the single cells were subjected to scRNA-seq.

Oscope has a broader scope than Cycler, says Liberali, but scRNA-seq data contain an inherent level of technical noise that can make the Oscope-based trajectories less accurate. Owing to the larger amount of information it can extract about expressed genes, Oscope is more suitable for identifying novel oscillating genes and correlating these oscillations with global patterns of gene expression, she says.

In Teichmann and Natarajan's view, the existing tools address different cell-cycle questions. For example, from any scRNAseq data set, Cyclone computationally assigns

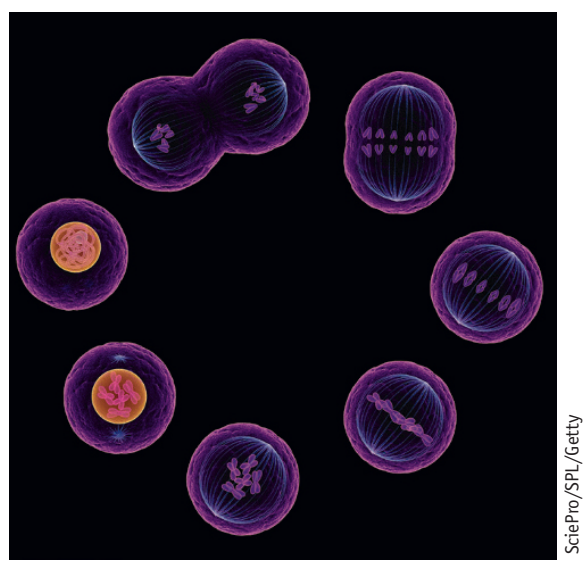

Cell division is crucial for many biological events, ranging from development and differentiation to cancer. 
cell-cycle stages to single cells; their scLVM uses highly variable genes across the cell cycle to identify correlated gene expression profiles. It identifies the cell-cycle contribution for a given cell type and for cells transitioning through processes such as development or differentiation, and helps discover distinct subpopulations. Oscope identifies oscillatory genes from single time points and orders cells on the basis of processes such as the cell cycle or responses to drugs or other stimuli. All of these methods can be applied to address similar questions, too, the researchers say, "but a comprehensive comparison has not yet been performed."

Both for human eyes and computational tools, it is tough to identify transitions between cell-cycle phases. That's true both in terms of gene expression and phenotype, says Liberali. To improve the method in that regard, she and her colleagues stained $S$ phase cells with EdU and noted that the peak of ERK activation at the G1/S transition was visible only when EdU staining was used. They have also improved Cycler by considering the variability of nuclear size in a cell population. Nuclear size is a proxy for progression through the cell cycle but also depends on how crowded the cells are, says Liberali. A cell in G2 in a crowded space has a smaller nucleus than a G1 cell in a sparser region of the population. In a single-cell gene-expression set, the technical variability is quite high, which makes it tough to set precise transitions between cell-cycle phases. Having few but very accurate descriptors of the transition is essential for the tool to yield precise data about transitions, says Liberali.

\section{Quiet, not silent}

Cells do not divide incessantly in the human body; they leave the cell cycle and become quiescent. Some researchers call these cells dormant but others bristle at that description. Quiescent cells are 'quiet' in terms of the cell cycle. In this phase, called G0, the cells are not replicating their DNA, but they are metabolically busy, says Kaldis, "they are not just sitting there."

Some cell types re-enter the cell cycle quickly, others might do so only after a few years, and others remain quiescent, postmitotic cells. Quiescent cells replenish adult cells and influence many types of cell-fate decisions. Understanding the early transitions from quiescence into G1 phase, as well as the appropriate signaling cues at a single-cell level, would explain how cellular memory is preserved and triggered for important cellular functions, say Teichmann and Natarajan. It's an area where single-cell genomics technologies will be helpful, they say.

Hilary Coller, a researcher the University of California, Los Angeles, studies how G0 is regulated and wonders whether there might be different types of quiescence, an idea that Kaldis has also pondered. Coller became intrigued by G0 after she did microarray analyses of gene expression changes in cells exposed to three different types of growth arrest for varying time periods. Experimentally, she and her team induce quiescence to compare them to proliferating cells. Their results suggest that quiescent cells are metabolically active, have a histone modification profile distinct from that of G1 cells and may be more reliant on the pentose phosphate pathway than proliferating cells.

Many patterns make quiescent cells distinct from G1 cells, says Coller. Quiescence appears to be dynamic; it is initiated, maintained and changed according to steps with differing gene expression patterns. The 'quiescence program' involves regulators of cell growth and division as well as genes that suppress apoptosis and differentiation and genes involved in cell-to-cell communication. In quiescent cells, there is also upregulation of tumor suppressor genes. Quiescent cells are not powered down; they can actively inhibit cell death and differentiation. Nevertheless, she says, some scientists argue G0 is but a continuation of G1.

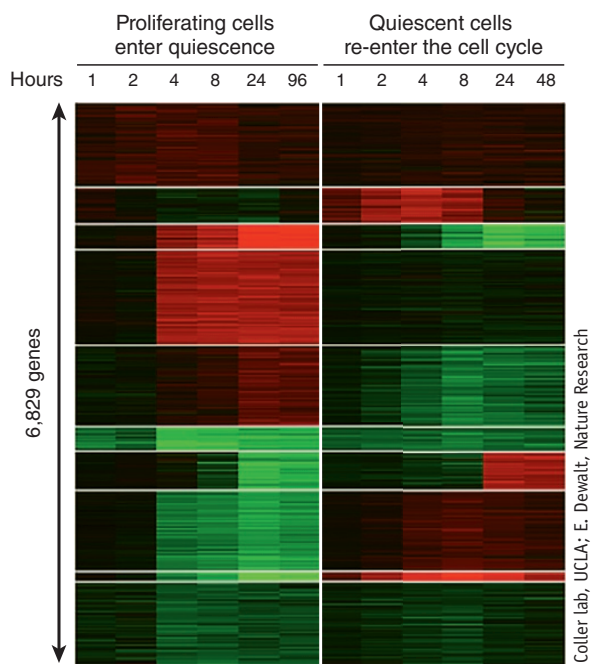

Widespread gene expression changes occur when proliferating fibroblasts enter quiescence (left) and when quiescent fibroblasts re-enter the cell cycle (right).

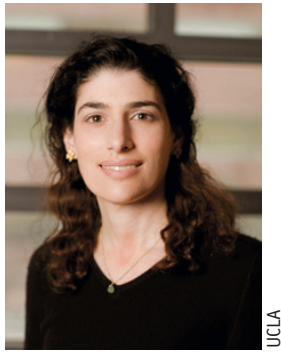

Quiescence sensors such as RIKEN's mVenusp $27 \mathrm{~K}^{-}$stand to help settle such questions. The team used gene expression data to validate sensor results, says Miyawaki.

There might be different types of quiescence, says Hilary Coller. But the researchers noticed that the sensor "cannot identify the G0 entry," which leads him and his team to regard it as "not perfect" just yet. Coller is interested in trying mVenus-p27 $\mathrm{K}^{-}$. To date, she and her team have not yet used the Fucci system, which she finds "powerful." She has been thinking about using the Fucci variant mVenus-p27 $\mathrm{K}^{-}$to distinguish G0 from $\mathrm{G} 1$. If done in live mice, for example, the entire cell cycle could be monitored in vivo.

In cancer, researchers long believed that proliferation was all that mattered, says Florian. That view led to the first generation of chemotherapy drugs. The fastestproliferating tissues in the human body are in healthy bone marrow and gut mucosa, says Florian. Leukemias can proliferate quickly, but, he says, “you'll see very, very few, if any, solid tumors proliferate at this rate." Some zones in a solid tumor proliferate little, others are necrotic, and others might comprise quiescent cells, which are usually resistant to chemotherapy.

Understanding the cell cycle and G0 has become crucial in cancer biology. Whereas it is relatively easy to kill proliferating cells with a drug, says Florian, "to find a drug that kills quiescent cells is very hard." Perhaps the new sensors will also help cancer biologists. More generally, these sensors let labs parse the cell cycle ever further, shining new light on the purportedly wellknown process of cell division.

\section{Vivien Marx is technology editor for} Nature Methods (v.marx@us.nature.com).

1. Sakaue-Sawano, A. et al. Cell 132, 487-498 (2008).

2. Oki, T. et al. Sci. Rep. 4, 4012 (2014).

3. Bajar, B.T. et al. Nat. Methods 13, 993-996 (2016).

4. Chittajallu, D.R. et al. Nat. Methods 12, 577585 (2015).

5. Scialdone, A. et al. Methods 85, 54-61 (2015)

6. Gut, G. et al. Nat. Methods 12, 951-954 (2015)

7. Leng, N. et al. Nat. Methods 12, 947-950 (2015). 